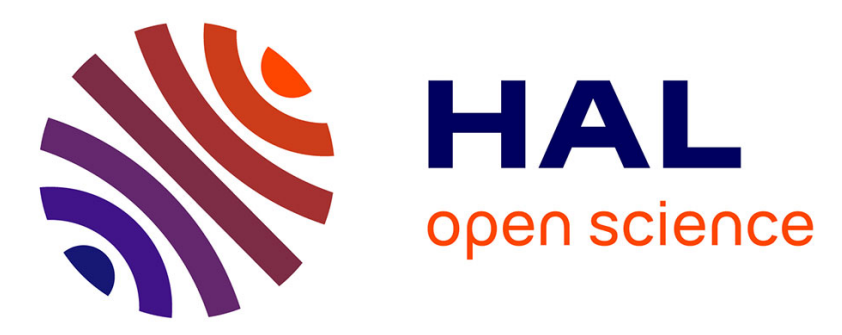

\title{
Soft Fault Diagnosis in Wire Networks Using Time Reversal Concept and Subspace Methods Soft
}

\author{
Moussa Kafal, Jaume Benoit, Andrea Cozza, Lionel Pichon
}

\section{To cite this version:}

Moussa Kafal, Jaume Benoit, Andrea Cozza, Lionel Pichon. Soft Fault Diagnosis in Wire Networks Using Time Reversal Concept and Subspace Methods Soft. International journal of digital information and wireless communications (IJDIWC), 2018, 8 (2), pp.85-89. 10.17781/P002411 . hal-01802225

\section{HAL Id: hal-01802225 \\ https://hal-centralesupelec.archives-ouvertes.fr/hal-01802225}

Submitted on 11 Dec 2020

HAL is a multi-disciplinary open access archive for the deposit and dissemination of scientific research documents, whether they are published or not. The documents may come from teaching and research institutions in France or abroad, or from public or private research centers.
L'archive ouverte pluridisciplinaire HAL, est destinée au dépôt et à la diffusion de documents scientifiques de niveau recherche, publiés ou non, émanant des établissements d'enseignement et de recherche français ou étrangers, des laboratoires publics ou privés. 


\title{
Soft Fault Diagnosis in Wire Networks Using Time Reversal Concept and Subspace Methods
}

\author{
Moussa Kafal ${ }^{*}$, Jaume Benoit*, Andrea Cozza ${ }^{\dagger}$, and Lionel Pichon ${ }^{\dagger}$ \\ ${ }^{*}$ CEA, LIST, F-91191, Gif-sur-Yvette, France \\ Email: moussa.kafal@cea.fr \\ ${ }^{\dagger}$ Group of Electrical Engineering - Paris (GeePs), CentraleSupelec, Université Paris-Saclay \\ 91192 Gif-sur-Yvette CEDEX, France \\ Email: andrea.cozza@centralesupelec.fr
}

\begin{abstract}
Time-reversal (TR) techniques recently adopted from acoustics to guided wave propagation along transmission lines have proven to be effectual in locating soft faults in different wire network configurations. In this paper, we apply the TR multiple signal classification (TR-MUSIC) technique to cable testing. TR-MUSIC is shown to provide spatial resolutions in the $\mathrm{mm}$ range, while using continuous-wave test signals. On the contrary, the well known time-domain reflectometry (TDR) methods would require large bandwidths in the order of gigahertz for a similar performance. The TR-MUSIC accuracy is demonstrated experimentally for locating both single and multiple soft faults in several cable networks.
\end{abstract}

\section{INTRODUCTION}

Despite the fact that huge efforts are being invested towards transforming the globe into a wireless world, most modern systems still rely on cables for energy and information distribution between their different parts. Surprisingly, statistics conducted on vehicles have shown that about 35\% of their total infrastructure is electrical based where a modern car can contain up to $4 \mathrm{~km}$ of wires. Add to that, several hundred $\mathrm{km}$ have cumulated in aircrafts which escalates in transport ships to more than $1200 \mathrm{Km} \mathrm{[1],} \mathrm{[2].} \mathrm{Consequently,} \mathrm{this}$ tremendous usage of cables with extensive long lengths is normally subject to aggressive internal and external conditions, namely manufacturing defects and environmental changes, etc., resulting in wire fault appearance.

These anomalies that affect a cable can be the origin of dysfunctions and imply serious consequences for the system or the environment. Generally, these wire defects can be categorized in two main families: hard or soft faults. Hard faults are typically short or open circuits which are the sources of many fires or signal losses. On the other hand, any fault which is not hard can considered a soft one, these include insulation damage, cracks, frays, etc. Notably, soft faults are characterized with weak reflectivities and are usually difficult to detect [3], [4]. Although, soft faults might not appear dangerous in their early stages, their potential degradation with time might end up with hard faults leading to catastrophic consequences. With this in mind, we focused our research efforts on dealing with such kind of faults due to their critically difficult detection, especially in complex wire networks [5][8].
Accordingly, guaranteeing a trustworthy usage of cables necessitates investing in the research and development of techniques qualified for precisely detecting the presence of faults in wire networks. Although, we have witnessed during the last few decades a wide range of methods employed for testing cables in an effort to locate faults, reflectometry kept the flagship in this domain [1], [9]. Originally developed in radar to locate targets and anomalies in free space, reflectometry injects a predefined testing signal into the network under test (NUT). The incident wave propagates according to the laws of propagation in the NUT until an impedance discontinuity is encountered (connector, junction, load or fault). Consequently, a reflection phenomenon will occur where a fraction of the incident energy will be reflected back to the injection point. The analysis of the received signal to the injected one provides very rich information about the presence, position, and nature of the impedance discontinuity. This information shall be valuable for maintenance operators to monitor the health of electrical networks and accordingly determine the degree of harmfulness this fault might bring to the whole system. Inspite the fact that promising results have been obtained when dealing with hard faults, difficulty in detection was encountered once soft faults are addressed [9]. Regrettably, the situation is expected to be worsened once branched networks are studied as multiple echoes from different impedance discontinuities in the system (junctions) might dwarf the one corresponding to the fault. Indeed, a similar effect is obtained once noise is encountered in the system.

With this in mind, it is worthy to note that a great resemblance exists between locating a soft fault in an NUT and detecting a target by radar in a cluttered environment. In fact, multiple scattering in the propagation medium can mask a weak target signature, jeopardizing its eventual detection. In other words, techniques used for target detection in a generic medium can be transposed to solve the problem of locating weak reflectivity soft faults in complex NUTs. Time-reversal (TR) concept developed in acoustics to retrofocus signals back to their source [10], allowed creating focal spots on a target's location simply by retransmitting a time-reversed version of echoes initially reflected by a target exposed to excitation signals.

Within this context, TR-based methods were adopted to 
solve the problem of detecting and locating soft faults in different complexity cable networks [5], [11], [12]. Notably, DORT method, a TR variant standing for decomposition of the TR operator, contributed significantly in the precise location of single and multiple soft faults in complex NUTs [6]-[8]. On the other hand, DORT necessitated testing the NUT over large bandwidths in the GHZ range to provide the conditions of a good spatial resolution which is not always available especially when dealing with band-limited systems. Besides, dealing with multiple faults in the same wire network required an iterative procedure integrated with the standard DORT method to provide accurate estimates of the fault locations [7].

For an efficient dealing with these problems, multiple signal classification (MUSIC) [13], a subspace method, succeeded efficiently in providing good estimates of targets' locations when applied in conjunction with TR processing. Markedly, the coupling between TR technique with the MUSIC algorithm has led to the creation of what is referred to as the TR-MUSIC [21]. This new concept has lead to a robust approach for locating targets in both homogeneous and non-homogeneous backgrounds especially in the cases of closely spaced targets or when using very sparse antenna arrays. This is true as the TR-MUSIC employs the null space of the multi-static data matrix (MDM) of the system. Accordingly, the TR-MUSIC based algorithm will be tested in this paper for estimating the locations of single and multiple soft faults in various cable network layouts.

\section{TIME-REVERSAL MUSIC}

Time reversal, is the process of emitting a time-reversed version of a source's captured response in open space so as to retrofocus around it. TR of acoustic waves has been applied with success to a wide variety of applications including medical imaging problems, non-destructive testing, target location in radar and sonar systems [10], [16]-[18], in addition to their compatibility with electromagnetic waves for the aim of target detection [19], [20]. Accordingly, TR-MUSIC will be adopted to guided wave propagation on transmission lines especially that it has lead to superesolution imaging using continuous wave excitations, a merit overshooting other methods in this domain.

\section{A. General Concept in open media}

In the last few decades, estimating the locations of pointlike scatterers in open space have caught enormous attention. For this purpose, Devany in [21] applied for the first time the MUSIC concept for the goal of estimating the location of targets from the scattered field data by employing subspace methods used in signal classification and discrete spectrum estimation.

In order to better understand the theoretical concept of TRMUSIC as applied in free space, let us consider an array of $L$ reversible transducers. In the first place, an inter-element impulse response (IEIR) expressing the received signals as functions of the transmitted ones is defined for each pair of transducers. For instance, the IEIR $k_{l m}(t)$ is the signal received on the channel number $l$ after a temporal Delta function is applied to the channel number $m$. According to the reciprocity theorem known for propagation in inhomogeneous medium, the IEIR from element number $m$ to $l$ is equal to that from $l$ to $m$, that is to say $k_{l m}(t)$ is equal to $k_{m l}(t)$. The repetition of the process for each pair $(m, l)$ of transmitting and receiving elements leads, at a given frequency, to the MDM or transfer matrix $\mathbf{S}(\omega)$ whose dimensions would be $L \times L$. It is worthy to note that all the data and signal processing is performed in the frequency domain and is associated with a single frequency $\omega$. Since all the expressions are associated with the given frequency, $\omega$ will be omitted from hereafter.

Since the TR operation is equivalent to a phase conjugation in the frequency domain, the TR operator (TRO), denoted as $\mathbf{K}$, can be defined as follows

$$
\mathbf{K}=\mathbf{S}^{\dagger} \mathbf{S}
$$

where $\dagger$ stands for the Hermitian transpose. In fact, the symmetry of $\mathbf{S}$ implies the TRO $\mathbf{K}$ is Hermitian, therefore $\mathbf{K}$ can be diagonalized and the number of significant eigenvalues hints to that of well-resolved scatterers. TR-MUSIC, like classical TR imaging as performed in DORT method, is designed to locate $M$ point targets from the knowledge of $\mathbf{K}$ under the condition that $L>M$ [20]. In fact, both DORT and TR-MUSIC are based on the eigenvalue decomposition of $\mathbf{K}$ where

$$
\mathbf{K}=\mathbf{U} \boldsymbol{\Lambda} \mathbf{U}^{\dagger}
$$

knowing that $\boldsymbol{\Lambda}$ is a real diagonal matrix of eigenvalues, and $\mathbf{U}$ is the eigenvector matrix. Notably, $\mathbf{U}$ can be decomposed into a signal space $\mathcal{S}$ and a noise or null space $\mathcal{N}$. Specifically, $\mathcal{S}$ is group of eigenvectors with the most significant eigenvalues, i.e., $\mathcal{S}=\operatorname{span}\left\{\mathbf{u}_{i}, \lambda_{i}>\lambda_{t h}\right\}$ whereas $\mathcal{N}$ is formed by the remaining eigenvectors having insignificant eigenvalues $\mathcal{N}=\operatorname{span}\left\{\mathbf{u}_{i}, \lambda_{i}<\lambda_{t h}\right\}$, given that $\lambda_{i}$ and $\mathbf{u}_{i}$ are the eigenvalues and their corresponding eigenvectors, respectively; $\lambda_{t h}$ is an arbitrary threshold determined by interpreting the eigenvalues of $\mathbf{K}$. For homogeneous and isotropic media, and in the framework of Born approximation [23], $\mathcal{S}$ and $\mathcal{N}$ are spanned by the conjugation of a quantity, such as a field or a voltage, generated by the $L$ transceivers; their interdependence can be modeled as a vector Green function $\mathbf{g}\left(X_{p}\right)$, with $X_{p}$ being the test location position in space. The locations of scatterers are found in TR-MUSIC imaging by computing a pseudo-spectrum $\Phi\left(X_{p}\right)$, defined with respect to the $\mathcal{N}$, as

$$
\Phi\left(X_{p}\right)=\frac{1}{\sum_{i}\left|\mathbf{u}_{i}^{\dagger} \mathbf{g}\left(X_{p}\right)\right|^{2}},
$$

where the sum is taken only over the $L-M$ indexes $i$ such that $\lambda_{i}<\lambda_{t h}$.

As a matter of fact, the orthogonality existing between $\mathcal{S}$ and $\mathcal{N}$ implies that the inner product $\mathbf{u}_{i}^{\dagger} \mathbf{g}\left(X_{p}\right)$ will deem to zero whenever the test location $X_{p}$ is equal to the actual position $X_{m}$ of one of the targets knowing that $m=1,2, \ldots, M$. 


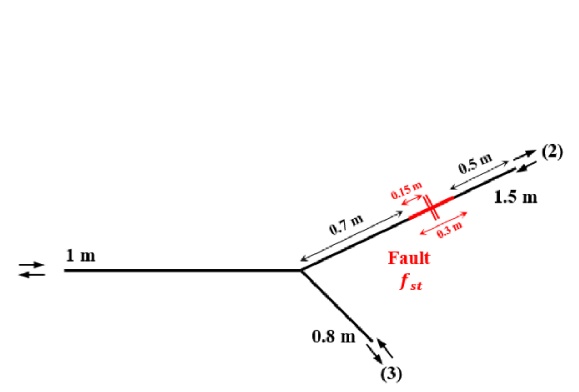

(a)

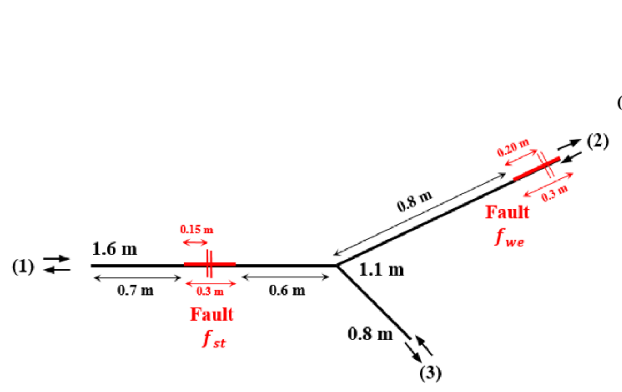

(b)

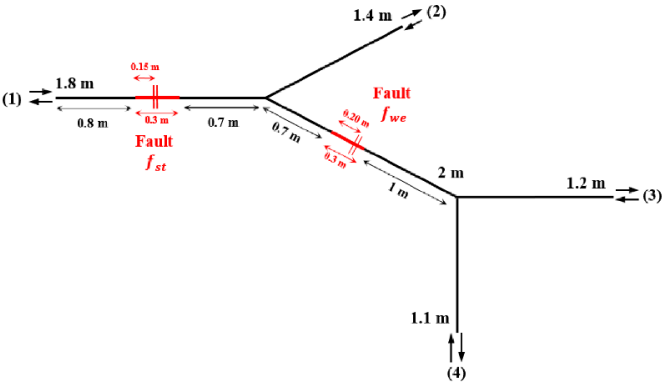

(c)

Fig. 1: Topologies of the cable networks used for the TR-MUSIC experimental validation: (a) with one soft fault; (b)\&(c) two soft faults with different intensities.

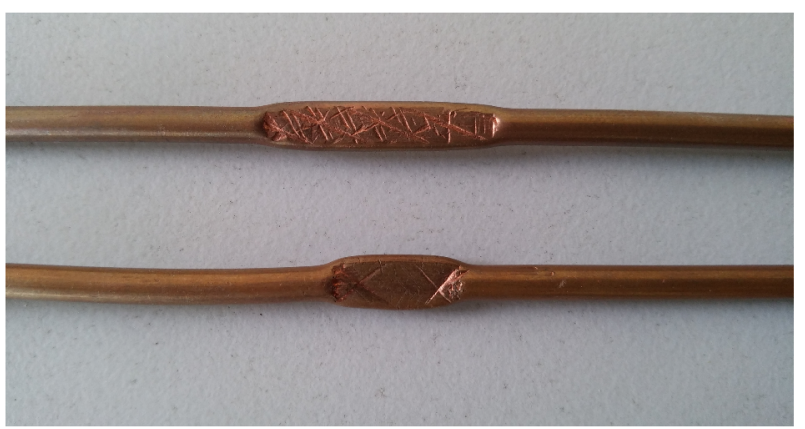

Fig. 2: Partially crushed semi-rigid coaxial cables used for creating the two faulty samples for the experimental validation of the TRMUSIC approach where the fault's reflection coefficient increases with an increase in the crushed area.

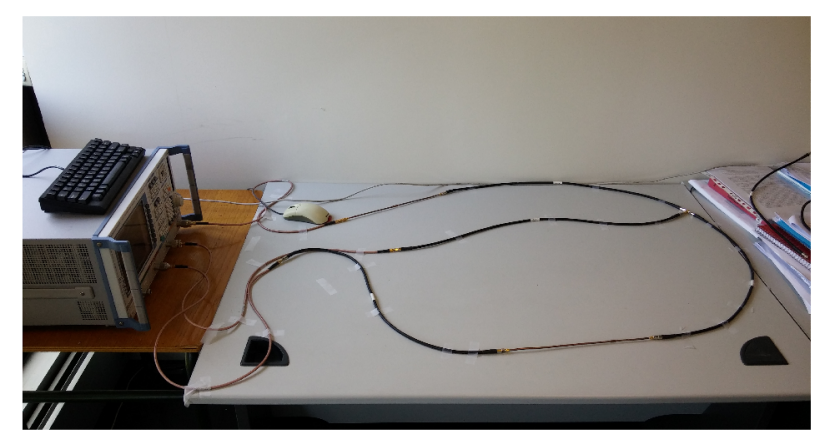

Fig. 3: The experimental setup of the double fault single Y-junction cable network of Fig. 1 (b), which shows the NUT's extremities connected to the vector network analyzer ZVB8 VNA.

Consequently, a singularity will be produced at each scatterer position which is basically obtained at each single frequency of the total applied bandwidth. Accordingly, a good spatial resolution of the scatterers' positions can be still acquired even using narrow bandwidths, an important feature provided by TR-MUSIC compared to other time domain fault detection techniques [6], [7], [24].

\section{B. Adoption to wire networks}

As mentioned earlier, a soft fault in an NUT can be seen as a scatterer in a generic medium. As a result, TR-MUSIC can be adopted to guided wave propagation along transmission lines in an effort to locate faults in wire network configurations. Markedly, the NUT's scattering matrix must be available to ensure the application of the method which can be obtained either by simulation or by measurement accomplished with a vector network analyzer (VNA), as will be done in sec. III.

It is important to note that assuring the applicability of fault detection and location methods has shown to be strongly associated with their integration with inverse problems namely the baselining approach [3], [5]-[7], [12], [25]. Perhaps, its importance becomes fundamental when dealing with soft faults as it will improve the signature and visibility of their weak reflectiveness especially in complex networks. Besides, it will produce a fault acting as a secondary source, an important feature necessary to derive the fault location approach used in time reversal (TR) based methods namely DORT and TRMUSIC as detailed in [5]-[8], [14].

The baselining procedure is composed of two main steps: first the scattering matrix $\mathbf{S}_{h}$ is measured for a non-faulty (healthy) version of the NUT, followed by another measurement on the same NUT after introducing the created soft faults producing $\mathbf{S}_{f}$. After that, the faulted and healthy scattering matrices are subtracted, giving rise to a baselined scattering matrix $\mathbf{S}=\mathbf{S}_{f}-\mathbf{S}_{h}$ which now contains mostly the contribution of the faults with negligible signatures of other components present in the NUT (junction, impedance mismatches, etc.). This matrix is then used in the TR-MUSIC procedure as described in the previous section, where computing pseudo-spectrum (3) requires an a priori model of the Green function of the medium. Specifically, for wire networks, a numerical simulator for transmission lines modeling the layout of the healthy NUT serves in retrieving the Green function which here relates the voltage at a position along the healthy NUT with the $N$ excitation voltages applied to its test ports. 


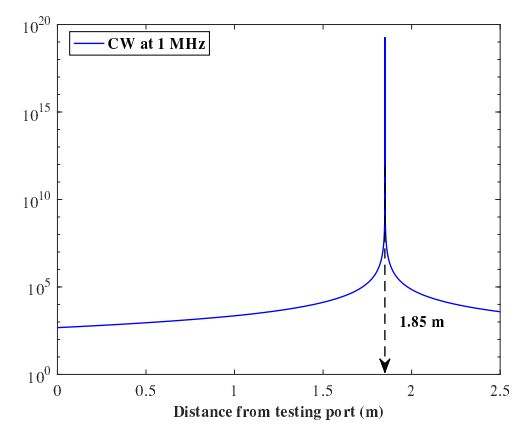

(a)

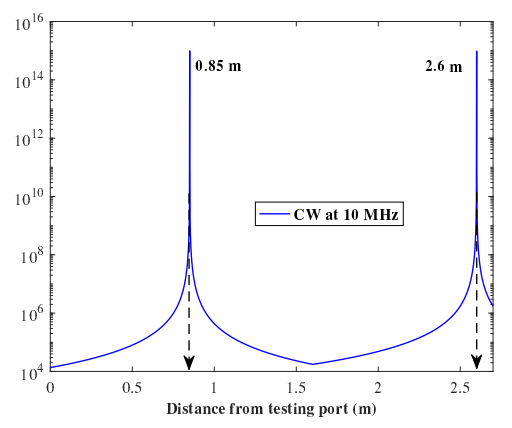

(b)

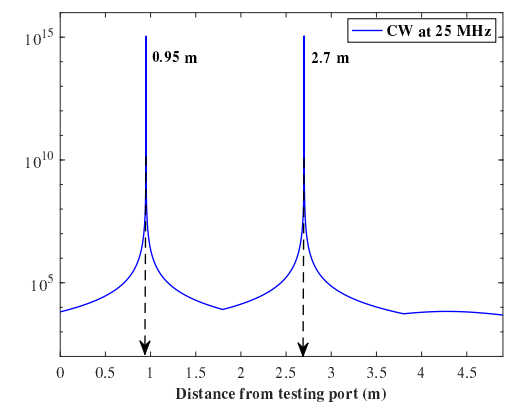

(c)

Fig. 4: Pseudo-spectra of the TR-MUSIC applied on the NUTs of Figs. 1(a),(b), and (c) computed on the basis of a continuous wave excitation of different frequencies. Super-resolved singularities were obtained on the faults' positions regardless the frequency used.

\section{RESUlts AND Discussions}

Experimental tests were conducted on two different complexity NUT layouts: a single and a double Y-junction network, which are affected by either a single or a double soft fault configuration as presented in Figs. 1(a)-(b)-(c). The NUTs are implemented using standard $50 \Omega$ coaxial cables which offer a practical advantage as faults can be inserted and removed in a controllable manner [6], [7]. Soft faults were introduced by applying a crushing force on a set of 3.4 $\mathrm{mm}$ cross-section, $30-\mathrm{cm}$ long semi-rigid coaxial lines. The crushed area determines the intensity of the soft fault, where the strongest fault $f_{s t}$ is obtained after applying a $2 \mathrm{~cm}$ crush while a $1 \mathrm{~cm}$ crushed area has led to the weaker fault $f_{w e}$ as shown in Fig. 2.

The extremities of each NUT served as testing ports by connecting them to a $300 \mathrm{kHz}$ to $8 \mathrm{GHz}$ Rohde \& Schwarz ZVB8 VNA, containing four testing ports where a complete setup shown in Fig. 3 serves as a clarifying example. After calibrating the VNA, studying the network consisted of measuring first the reference healthy scattering matrix $\mathbf{S}_{h}$ of the NUT without the faults which was accomplished by using the unaltered $30-\mathrm{cm}$ semi-rigid sections. This was followed by replacing the unaltered cable samples with the faulty ones and measuring the faulty scattering matrix $\boldsymbol{S}_{f}$ of the NUT. After that, the procedure described in sec. II is applied. The imaging pseudo-spectrum of each NUT is computed by means of an in-house transmission-line solver, implemented under Matlab.

The single Y-junction NUT of Fig. 1 (a), containing a single soft fault at $1.85 \mathrm{~m}$ from the reference testing port (1) is firstly considered. The corresponding scattering matrices were measured based on a monochromatic pulse (single tone, no bandwidth) of frequency $1 \mathrm{MHz}$. Fig. 4 (a) shows the pseudospectrum computed from the experimental data; the distances on the horizontal axis are measured from port (1), as defined in Fig. 1. Remarkably, an accurate estimate is obtained on the fault's position, where the singularity attained illustrated in Fig. 4 (a) unveils the main advantage TR-MUSIC has introduced in the domain of fault detection and location along transmission lines. As a matter of fact, it has shown its ability to provide a good spatial resolution at the fault's position while using a single frequency excitation.

In order to check the method's applicability with more complex NUTs, TR-MUSIC is examined on the single and the double Y-junction networks of Figs. 1 (b)\& (c) containing two faults each. Significantly, super-resolved singularities are obtained on their position as demonstrated in the pseudospectra of Figs. 4 (b)\& (c) when measured using a $10 \mathrm{MHz}$ and $25 \mathrm{MHz}$ continuous waves respectively. It is also important to realize, that the results obtained are not affected by the frequency of the excitation wave, as long as it is below a maximal frequency condition detailed in [14], a prominent feature proving the method's effectiveness in locating soft faults based on continuous single frequency waves. Eventually, this demonstrates the technique's applicability in band limited systems without the need of complex testing setups requiring pulse generators and fast electronics. Markedly, another important feature has been unleashed which is designated by the feasibility of TR-MUSIC in locating single as well as multiple soft faults in different network complexities based on monochromatic measurements accompanied with a subwavelength spatial resolution.

\section{CONCLUSIONS}

This article has shown once again that TR methods could be applied to locate faults in complex cable networks which was demonstrated here by the TR-MUSIC method. It has been shown experimentally the feasibility of this technique in detecting and locating single and multiple soft faults in two NUTs with increasing complexity. The outstanding performance of TR-MUSIC in ensuring a sub-millimeter resolution while utilizing relatively low test frequencies is an extraordinary feature, since obtaining such performance with TDR techniques is impossible unless higher test frequencies and wider bandwidths are used. Regrettably, this might be easily obstructed by attenuation in cables. Indeed, the most striking feature of TR-MUSIC is that it ensures sub-wavelength spatial resolution in the $\mathrm{mm}$ range while using continuous signals. 


\section{REFERENCES}

[1] C. Furse and R. Haupt, "Down to the wire [aircraft wiring]," Spectrum, IEEE, vol. 38, no. 2, pp. 34-39, 2001.

[2] F. Auzanneau, "Wire troubleshooting and diagnosis: Review and perspectives," Progress In Electromagnetics Research B, vol. 49, pp. 253 279, 2013.

[3] L. A. Griffiths, R. Parakh, C. Furse, and B. Baker, "The invisible fray: A critical analysis of the use of reflectometry for fray location," Sensors Journal, IEEE, vol. 6, no. 3, pp. 697-706, 2006

[4] A. Cozza and L. Pichon, "Echo response of faults in transmission lines: Models and limitations to fault detection," IEEE Transactions on Microwave Theory and Techniques, vol. 64, no. 12, pp. 4155-4164, 2016.

[5] L. Abboud, A. Cozza, and L. Pichon, "A matched-pulse approach for soft-fault detection in complex wire networks," Instrumentation and Measurement, IEEE Transactions on, vol. 61, no. 6, pp. 1719-1732, 2012.

[6] L. Abboud, A. Cozza, and L. Pichon, "A noniterative method for locating soft faults in complex wire networks," Vehicular Technology, IEEE Transactions on, vol. 62, no. 3, pp. 1010-1019, 2013.

[7] M. Kafal, A. Cozza, and L. Pichon, "Locating Multiple Soft Faults in Wire Networks Using an Alternative DORT Implementation," Instrumentation and Measurement, IEEE Transactions on, vol. 65, no. 2, pp. 399-406, 2016.

[8] M. Kafal, A. Cozza, and L. Pichon, "An efficient technique based on dort method to locate multiple soft faults in wiring networks," IEEE Instrumentation \& Measurement Magazine, vol. 19, no. 4, pp. 10-14, 2016.

[9] C. Furse, Y. C. Chung, R. Dangol, M. Nielsen, G. Mabey, and R. Woodward, "Frequency-domain reflectometry for on-board testing of aging aircraft wiring," Electromagnetic Compatibility, IEEE Transactions on, vol. 45, no. 2, pp. 306-315, 2003.

[10] M. Fink and C. Prada, "Acoustic time-reversal mirrors," Inverse problems, vol. 17, no. 1, p. R1, 2001.

[11] R. Razzaghi, G. Lugrin, H. Manesh, C. Romero, M. Paolone, and F. Rachidi, "An efficient method based on the electromagnetic time reversal to locate faults in power networks," IEEE Transactions on Power Delivery, vol. 28, no. 3, pp. 1663-1673, 2013.

[12] L. El Sahmarany, L. Berry, N. Ravot, F. Auzanneau, and P. Bonnet, "Time reversal for soft faults diagnosis in wire networks," Progress In Electromagnetics Research M, vol. 31, pp. 45-58, 2013.

[13] R. O. Schmidt, "Multiple emitter location and signal parameter estimation," Antennas and Propagation, IEEE Transactions on, vol. 34, no. 3 , pp. 276-280, 1986.

[14] M. Kafal, A. Cozza, and L. Pichon, "Locating Faults with High Resolution Using Single-Frequency TR-MUSIC Processing," IEEE Transactions on Instrumentation and Measurement, vol. 65, no. 10, pp. 2342 2348, 2016.

[15] F. K. Gruber, E. A. Marengo, and A. J. Devaney, "Time-reversal imaging with multiple signal classification considering multiple scattering between the targets," The Journal of the Acoustical Society of America, vol. 115, no. 6, pp. 3042-3047, 2004.

[16] M. Fink et al., "Time-reversed acoustics," Scientific American, vol. 281, no. 5, pp. 91-97, 1999.

[17] Y. Hristova, P. Kuchment, and L. Nguyen, "Reconstruction and time reversal in thermoacoustic tomography in acoustically homogeneous and inhomogeneous media," Inverse Problems, vol. 24, no. 5, p. 055006 , 2008.

[18] W. Zhang, A. Hoorfar, and L. Li, "Through-the-wall target localization with time reversal music method," Progress In Electromagnetics Research, vol. 106, pp. 75-89, 2010.

[19] C. Prada and M. Fink, "Eigenmodes of the time reversal operator: A solution to selective focusing in multiple-target media," Wave motion, vol. 20, no. 2, pp. 151-163, 1994.

[20] A. J. Devaney, "Super-resolution processing of multi-static data using time reversal and music," J. Acoust. Soc. Am, 2000.

[21] A. Devaney, "Super-resolution processing of multi-static data using time reversal and music. submitted to j," Acoust. Soc. Am, 2001.

[22] W. GAO, X. WANG, and B. Wang, "Review of time reversal imaging techniques."

[23] M. Born and E. Wolf, Principles of optics: electromagnetic theory of propagation, interference and diffraction of light. Cambridge university press, 1999.
[24] S. J. P., Time-domain Reflectometry for Monitoring Cable Changes: Feasibility Study. Electric Power Research Institute, 1990.

[25] G. Cerri, R. De Leo, L. Della Nebbia, S. Pennesi, V. M. Primiani, and P. Russo, "Fault location on shielded cables: Electromagnetic modelling and improved measurement data processing," IEE Proceedings-Science, Measurement and Technology, vol. 152, no. 5, pp. 217-226, 2005. 